InVisible Culture Journal • Issue 30: Poetics of Play

\title{
Wait Wait... Don't Play Me: The Clicker Game Genre and Configuring Everyday Temporalities
}

Oscar Moralde ${ }^{1}$

${ }^{1}$ UCLA

Published on: Apr 18, 2019

DOI: $10.47761 / 494 a 02 f 6.0 c f 44974$

License: Creative Commons Attribution 4.0 International License (CC-BY 4.0). 


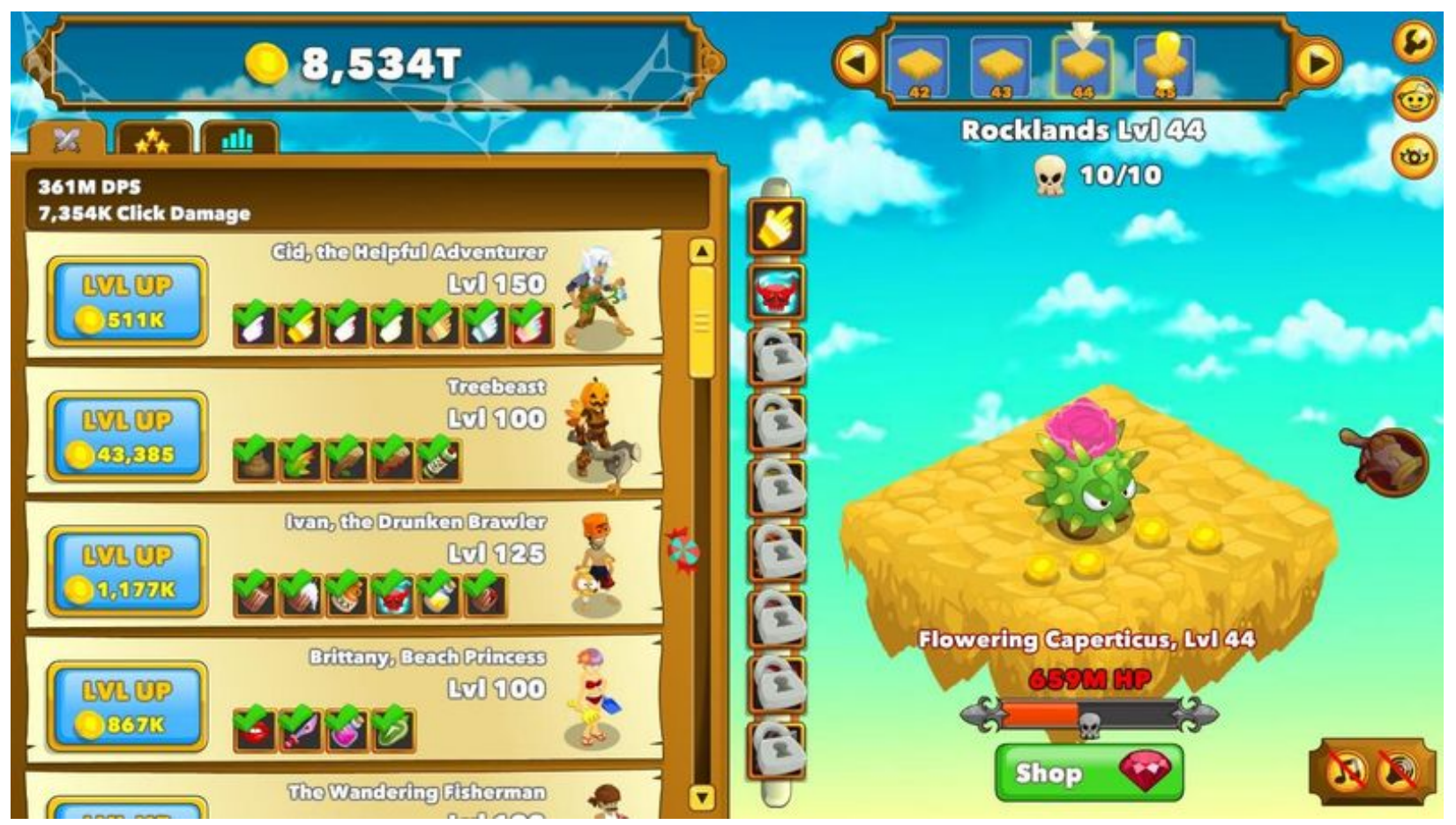

Clicker Heroes, 2014.

"We do not say that we have learnt, and that anything is made new or beautiful by mere lapses of time; for we regard time itself as destroying rather than producing, for what is counted in time is movement, and movement dislodges whatever it affects from its present state." $\underline{1}$

“The Time Machine brings cookies from the past, before they were even eaten." $\underline{2}$

\section{Game Genre, Duration, and the Flow of the Everyday}

Video game aesthetics extend beyond the sights and sounds encoded into datasets for electronic processing into the audiovisual worlds of player experience. They even extend beyond the feel and feelings produced by the cybernetic intersubjective assemblage of player and game at the threshold of the interface, which has become an important site of inquiry for game studies scholars. $\underline{3}$ Game aesthetics are strongly situated aesthetics: spatial and temporal contexts not only shape the meanings that players take away from gameplay experience, but they also determine the form and types of experience that unfold in play. For example, in Hamlet on the Holodeck, Janet Murray describes the iconic 1984 puzzle game Tetris as "a perfect enactment of the 
overtasked lives of Americans in the 1990s-of the constant bombardment of tasks that demand our attention and that we must somehow fit into our overcrowded schedules and clear off our desks in order to make room for the next onslaught... Tetris allows us to symbolically experience agency over our lives." $\underline{4}$ This passage has been cited as a historical flashpoint for the so-called ludology-narratology debates and on related questions of the value of symptomatic readings of video games as texts-whether the audiovisual and gameplay elements that constitute Tetris signify a coherent critique, the importance of elaborating that critique, and other similar questions. $\underline{5}$

However, the profound insight that Murray offers with this example is not solely contained in what Tetris is, but in delineating how, where, and when Tetris might be played. Her reading of the game does not assume an idealized abstract concept of a player but a specific playing subject: in this case, a worker inhabiting an everaccelerating regime of labor that places increasing demands on the subject's time and attention. This specific configuration of everyday life is the ground against which Murray examines the game's qualities; those qualities, because of how they use and shape time, make a good fit (so to speak) for such a life. The core gameplay "loop" of Tetris, which involves placing a series of blocks into proper places in order to clear lines of blocks off the board until the accelerating inputs clutter the board too quickly and end the game, does more than evoke the feeling of task management through its assembly-line game mechanics. Those relatively straightforward mechanics also work well in short play sessions of a few minutes where the player starts from scratch each time. Thus, the mechanics not only resemble task management, but also cohere into experiences that fit into the gaps of a player's everyday schedule of real-life tasks; the game itself takes on the temporality of a task by structuring and capturing otherwise unordered time. Murray's insight, which links gameplay experience to everyday life, raises a key question for video game aesthetics: how do specific game design choices and gameplay forms address everyday temporalities? What kinds of lived experience do games envision, and how do they fit into that experience?

The aesthetics of duration (along with intermittence and repetition) within specific reception contexts is not unique to video games; investigations into the mode of bingewatching television series on streaming platforms, or the durational experience of slow cinema within theatrical and museum contexts, address similar questions about how formal-aesthetic properties of a work shape how it fits into one's lifeworld. $\underline{6}$ What is arguably specific to games, however, is how duration binds to questions of genre and the expectations that players have about game genre; these questions tie to the 
protean status of game genre classification in general. For example, in cinema studies Steve Neale suggests genre as a relational process between audience expectations and an historicized corpus of films dominated by specific aesthetic strategies such as spectacle, and Linda Williams has written of the embodied reactions that are implicated in classifying genres such as horror, pornography, and melodrama. $\underline{7}$ Yet, these concerns remain relatively agnostic about duration and are portable across short films, two-hour features, and long-form series-slow cinema as a quasi-genre category derives some uniqueness from its inordinate reliance on duration compared to most genre classifications.

In the study of game genre, the relationship between audience expectations, dominant aesthetic strategies, and embodied reactions remains critical, but is further complicated by frameworks of player experience. Thomas H. Apperley has criticized conventional and popular genre categories for relying on "loose aesthetic clusters based around video games' aesthetic linkages to prior media forms," while Dominic Arsenault advocates discarding static genre models by noting that "the genre of a game is tied not to an isolated, abstracted checklist of features, but to the phenomenological, pragmatic deployment of actions through the gameplay experience." 8 Such a deployment of action for a given genre is strongly tied to the experience of duration. An abstract dexterity-based puzzle game like Tetris is usually experienced through discrete self-contained minutes-long play sessions, while a competitive fighting game such as Street Fighter $V$ (2016) might segment into even quicker bouts of 99 seconds. Call of Duty (2003) and other contemporary first-person shooter games have single-player campaigns of about six to eight hours, while a roleplaying game such as The Witcher 3 (2015) can last for dozens or even hundreds of hours of play. While there are certainly experiments and innovations in each genre that defy these expectations, both Neale and Arsenault note that genre hybrids and outliers rely on intuitive understandings of the genre's conventions to distinguish themselves. $\underline{9}$ If some of these conventions attend to duration, then identifying or creating a new game genre also creates new possibilities for understanding temporal experience.

Game studies scholars have long been fascinated with the fragmented, amorphous, elastic, and recursive nature of game time that is built into the disjunction between human and computer temporalities, and the myriad forms of temporal manipulation that include pauses, save states, slow motion, replays, and rewinding. In Game Time: Understanding Temporality in Video Games, Christopher Hanson astutely analyzes and synthesizes a wide range of theoretical models of video game time "to argue that what 
fundamentally differentiates the temporal structures of video games from other media is their unique engagement with liveness, causality, potentiality, and lived experience." 10 While Hanson primarily focuses on aesthetic strategies and mechanisms within games that structure the navigation and experience of temporality, he gestures toward the connection between games and broader considerations of lived time in a brief consideration of mobile games, writing in his conclusion: "The ways in that mobile games have become part of the daily lives of millions of people demonstrates the surprising and pervasive manner that game time has become part of lived experience." 11 That is, players actively place the experience of a specific game into the larger temporal context of everyday life.

Building from that discussion, I examine that ever-changing placement through the lens of a young game genre that plays on the tension between player-game temporalities: the clicker game, also known as the idle game, the incremental game, or the background game. The clicker exemplifies Murray's vision of endless multitaskingas-game, updated and accelerated for the technocultural landscape of the 2010s. In its fragmentation and intermingling of temporalities, it both dramatizes and enacts a technocratic regime of time-sharing that is endemic to contemporary neoliberalism's colonization of ever more granular units of time. In its aesthetic ambiguity, the clicker genre is symptomatic of the absolute conflation of time and monetary value, yet also allows for the possibility of contemplating and experiencing resistance to that process -to find some time of one's own. However, fully explicating the significance of clicker aesthetics requires contextualizing the genre within the cultural spaces of social media and mobile games, the structures of its gameplay mechanics and themes, and the technical capabilities of the hardware platforms it inhabits.

\section{Rise of the Clicker Genre and Waiting-as-Gameplay}

In 2015, game critic Jeff Gerstmann offered one provisional definition of the clicker genre based on its mechanics:

These are games where you click on things to watch numbers go up, and eventually in many of these games you are able to hire things that effectively click for you. You are then earning more currency to spend on more hirelings that click on more things for you. So the numbers go up faster. It becomes harder to kill the things you need to click on-it's often a combat scenario but not always. It's this exponential machine that never necessarily gets easier or harder. The numbers just keep going up... [emphasis added] $\underline{12}$ 
Brendan Keogh and Ingrid Richardson offer their own definition of what they call "background games," which include features such as "the need to complete regular repetitive tasks to achieve long-term larger goals" and enforced waiting periods where the player leaves the game process running in the background before returning to play minutes, hours, or even days later. $\underline{13}$ This more expansive definition, which focuses primarily on the duration and intermittence of play, traces strong affinities between these games and discussions of casual games often found on mobile platforms. It includes games such as The Simpsons: Tapped Out (2012) which have arguably "more" gameplay than typical clickers, but it is productive to consider the relative quantity of gameplay in the clicker genre, and the centrality Keogh and Richardson assign to waiting as player action make their findings vital to this discussion. Alharthi et al. have crafted one of the most rigorous definitions for the genre to date, stating that idle games are "games that can progress without player interaction for some period of time" and which "involve clicking or tapping to set up a game, to generate resources, and to spend resources on upgrades." $\underline{14}$ They identify a subset of idle games called incremental games, which include features that automate the clicking process and often model an internal economy. In this framework, idle games are a separate category from clicker games, which are defined as "any in which the player clicks a button for a reward," making incremental games an overlap between the categories of idle game and clicker game. $\underline{15}$

These distinctions are rarely made in the popular discourse, and the instability of the nomenclature reflects the relative youth of the genre. For example, instances of these games on phones are often called "tap games," such as Tap Titans (2014), because of how they use the touchscreen interface. However, because of the prominence of the personal computer within games discourse and the significance accorded to Ian Bogost's Cow Clicker (2010) and Julien “Orteil” Thiennot's Cookie Clicker (2013) as some of the earliest examples, "clicker game" is often used interchangeably with "idle game" as a catch-all genre classifier, as seen in a 2015 headline announcing "Clicker Games Are Suddenly Everywhere On Steam.”트 I am less interested in stabilizing naming conventions or parsing subcategory boundaries than I am in, per Neale, discerning the dominant or dominating aesthetic that centripetally binds these games into a legible grouping. In this case, the key aspect to consider here and across all these definitions is the concept of waiting transformed into gameplay action: not actively playing on a moment-by-moment timescale is incorporated into, or even required for, gameplay progress across a longer timescale. 
The names of traditional game genres emphasize the action that unfolds within the gameworld, such as fighting, shooting, or role-playing. As names, both "clicker" and "idle" try to parse the absence of those commonly understood modes of action, either by foregrounding the seemingly-passive non-action of waiting, or the minimalist physical motion of clicking that structures that waiting. In their interfaces and theming, many clicker games resemble established genres such as city-building simulations or real-time strategy games. However, unlike those genres, in which the gameworld simulation can continue to run without constant player input but requires that input to progress, within clicker games the player's input becomes less and less integral to progress over time. While the player's initial clicks might make significant, meaningful progress towards the game's end goal, the "exponential machine" structure makes the five hundredth, or five thousandths, click less so. In most clicker games, the player's initial clicks directly "make the numbers go up" far more quickly than the game's automated processes; but as the game progresses, the situation is reversed, and the game thus encourages the player to focus on indirectly increasing the rate at which the numbers go up by augmenting the automation. Constant clicks give way to intermittent intervention, or as Alharthi et al. state, "play less and plan more." [emphasis in original] $\underline{17}$ The trajectory of a clicker game is to find better ways to not click.

This focus on absence means that "clicker" as a genre signifier offers instructive parallels with the "walking simulator" genre: both names originated as ironic judgments on the supposed lack of meaningful gameplay within these games by centering on the action verb that was the closest thing to "real" play. However, the terms lost most of their pejorative quality as more games earnestly embraced the experiences associated with each and thus consolidated into a legible grouping. Melissa Kagen notes that the term "walking simulator" was meant to mock games such as Dear Esther (2012) and Gone Home (2013), which enact first-person traversal of a 3D environment but without traditional gameplay elements like combat or puzzlesolving, as one would find in established genres like the first-person shooter or adventure game. The assignment of a new genre label as a badge of difference became a politically charged act amidst the contemporaneous Gamergate movement which attempted to police the boundaries of "gamer culture" by antagonizing game developers, journalists, and communities they considered outside the culture.

Gone Home, for example, used its walking mechanics to explore feminist and queer themes through its narrative, an intersection which made the game a target. Kagen writes, "Between its pleas to keep politics out of gaming and its association of feminist 
politics with walking sims, the \#GamerGate faction tied together the fear that games were becoming unrecognizable to gamers with the fear that women were taking over nerdy male spaces." 18 Calling these games "walking sims" was one way to exclude them, and the people associated with them, from that culture. However, developers and critics reclaimed the term and began using it themselves as games in that vein continued to proliferate and find an audience. These games provided a distinct set of experiences from first-person shooters and adventure games, and the term already had public recognition, making it a useful identifier for players who sought out those experiences. Reviews and discussions of walking sims now use the term freely, though often still with some ambivalence and acknowledgment of its contested origins. $\underline{19}$

The clicker genre has traced a similar cultural trajectory to the walking sim. In his podcast discussion, Gerstmann provides an accurate description of the clicker genre, but he frames it somewhat comedically by self-consciously wrestling with the idea that his earnest enjoyment of these games might be absurd or pathological. Aubrey Anable has discussed the gendered perception of casual phone games, where some groups within gaming culture dismiss the majority audience of women playing them as not playing real games, because they lack the "procedural complexity, expensive hardware, and graphic realism" associated with dominant modes of game production and consumption. $\frac{20}{}$ Clickers also lack those technical characteristics, but they have generally avoided such gendered dismissal through ironic distancing strategies, such as tapping into the absurdity that Gerstmann's discussion brings to the fore.

The clicker genre has antecedents such as virtual pets and the satirical "zero-player games" Progress Quest (2002) or StatBuilder (2008), which Staffan Björk and Jesper Juul argue are "appreciated more as artifacts with potential activities than as actual activities enabled through these artifacts. We are not expected put considerable time into playing these games, but rather to consider them as hypothetical experiences." $\underline{21}$ But the first game to popularize the term "clicker," and to perhaps elicit the expectation of actually putting considerable time into playing, was Ian Bogost's Cow Clicker (2010). With its core gameplay of clicking an image of a cow every six hours to make a number go up and the possibility of accelerating that rate of increase by inviting Facebook friends and spending real money, Bogost intended for the game to satirize the predatory design practices of social media games such as Zynga's popular Facebook game Farmville (2009). $\underline{22}$ While lacking the antagonistic exclusionary intent carried by those who used "walking sim" as a derogation, the rhetoric of "clicking" within Cow Clicker as minimalist action makes a similar kind of normative claim about 
what should constitute meaningful gameplay by focusing on its supposed absence. However, this claim was upended by the thousands of players who became emotionally invested in their cows, and who responded with anguish when Bogost ended the parodic experiment with a “Cowpocalypse” in September 2011. 23

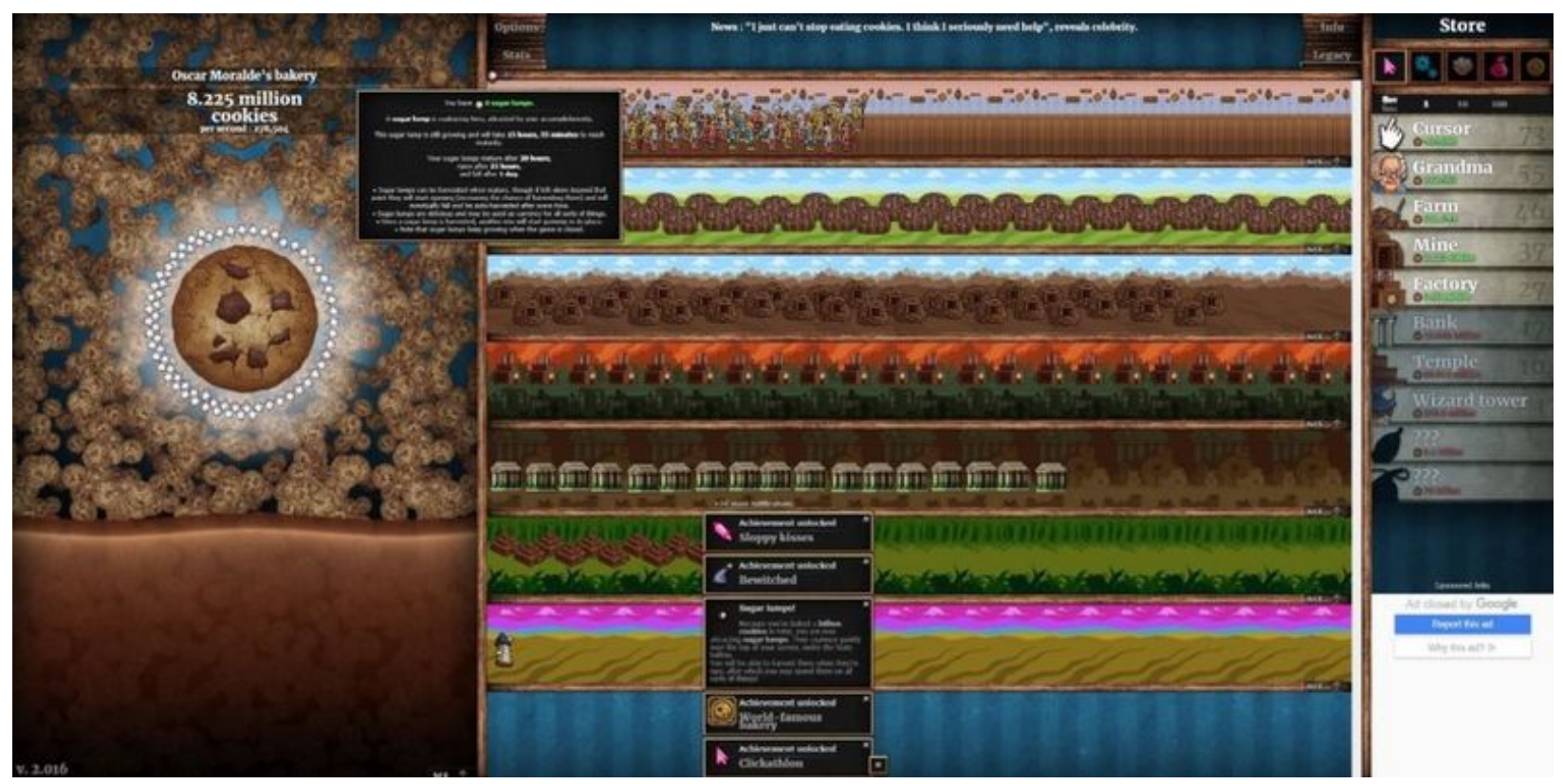

Figure 1. Julien "Orteil" Thiennot, Cookie Clicker, 2013, video game.

"Clicker" became cemented as both a grouping of games and a term to classify them with Julien "Orteil" Thiennot's Cookie Clicker (2013), which established the template for many subsequent clicker games (figure 1). Bogost acknowledges the game as "the logical conclusion to Cow Clicker, the place I didn't think to take it: a game you don't even have to play... For whatever reason, the conditions were right for not only one or two clicker-games, but a whole genre of them." $\underline{24}$ While characterizing the action of Cookie Clicker as not-even-playing again makes a restrictive normative claim about the nature of gameplay, he is correct in describing the game as both an extension of Cow Clicker and as a genre template. Cookie Clicker establishes a pattern that many subsequent clicker games follow: the player clicks on a giant cookie to produce cookies. The number of cookies is also used as a currency to purchase upgrades that automate cookie production, initially through hiring grandmas that bake cookies for you and constructing cookie factories, up to constructing wizard's towers and dimensional portals to produce quintillions and quintillions of cookies through magic and superscience. As the player increases their cookie net worth and production rate, they also unlock secondary currencies and other markers of progress. They can also choose to reset the game, returning their cookie count to zero but retaining a 
permanent boost to their automated cookie income based on how many cookies they sacrifice, thus making the upward climb quicker on subsequent attempts.

In the past several years, this template has inspired many variations on the theme. Some clicker games provide novelty in their visuals or settings: Clicker Heroes (2014) uses the premise of fantasy heroes fighting powerful monsters (figure 2). Time Clickers (2015) recasts its clicks as lasers chipping away at large blockish enemies in an abstract grid-like battlefield, and Trimps (2015) lets the player develop a society of small imp-like creatures-yet all use a very similar gameplay loop to Cookie Clicker. Some of the more intriguing clicker games use the premise to evoke stories and themes. Succubox (2017), for example, launches a Cow Clicker-like satirical attack on the video game business practice of selling virtual "loot boxes" for real currency (figure 3). Megalomaniac (2016) draws a broader capitalist critique by putting the player in the role of a billionaire heir whose inherited and invested wealth grows exponentially without even trying. Spaceplan (2016) puts the player on a space probe and charts the slow process of exploring and colonizing a new planet, with a snarky onboard computer providing commentary along the way (figure 4).

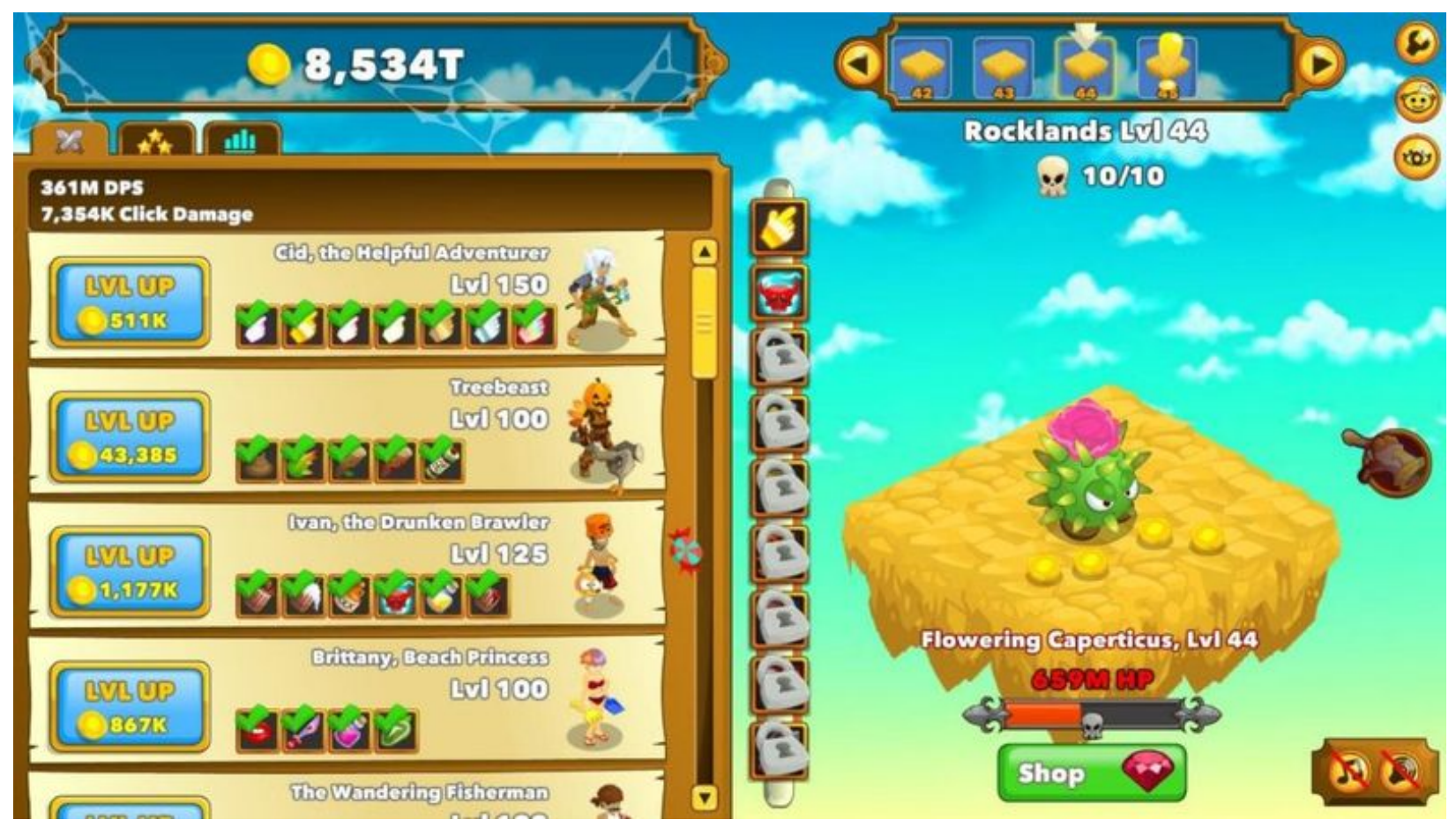

Figure 2. Playsaurus, Clicker Heroes, 2014, video game. 


\section{SHOP}

Fulfillment (100 Levels)

You can finally stop playing.

Figure 3. Tyler Glaiel, Succubox, 2017, video game.

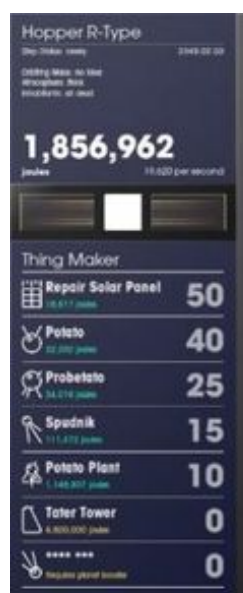

Figure 4. Jake Hollands, Spaceplan, 2017, video game. 
As some of these descriptions evoke, many clicker games use humor and absurdity as conventional elements. In part, designers must thematize the mechanical process of numbers growing exponentially larger in ways that make relative sense to the player; thus, the progression from grandmas to dimensional portals in Cookie Clicker. But the core experience of the genre-waiting and seemingly "doing nothing"-itself produces aesthetic tension and incongruity. The progression curve of the clicker genre ensures that as a player spends more time with the game, they actively click less and computer automation "clicks" more; against this curve, absurdity is a reaction to the implicit question of why a player would find this game enjoyable and play it for the long durations required to see progress.

One explanation for the genre's prevalence often invoked in discussing popular mobile or casual games, is that the gameplay mechanics prey on psychological compulsion and addiction. $\underline{25}$ In the case of some clicker games, this is true; they contain pay-to-win mechanics where players can spend real money to decrease wait times or convert it to in-game currency, and those games' growth curves can be designed with breakpoints where progress slows enough to tempt players. $\frac{26}{}$ Bogost intended to satirize these regressive kinds of design choices with Cow Clicker, only to find people that were

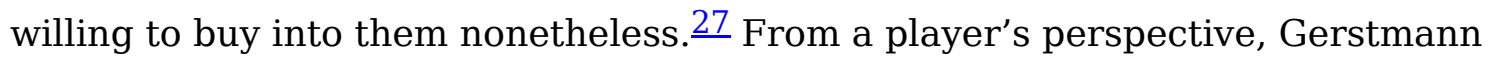
describes a compulsion to play clickers until he "breaks" himself from the game by cheating and artificially inflating the numbers; after stepping outside the rules of the game and making the numbers meaningless to him, he loses the desire to continue playing. But while the possibility for compulsion and exploitation exists, the genre does not require monetization, and many clickers do not offer real-money microtransactions. Many fans of clicker games will reject overly exploitative games where paying real money is the only pathway for progress. In addition, Keogh and Richardson interviewed players of background games and found that many derived satisfaction from rejecting the option to pay for advancement in favor of waiting the allotted time for the numbers to go up on their own-the opportunity to pay and the choice to reject it became one part of the gameplay. $\underline{28}$

One of the binding factors of genre is how it guides audience expectations, such as through a focus on specific themes or narrative tropes. In the case of clickers, their exponential mechanisms often critique the logic of capitalist accumulation: Gerstmann associates clickers with "corporate culture," while Keogh and Richardson link background games to "neoliberal labor and gamified workplaces." 29 Clickers also often cast doubt on utopian assumptions about the value of automation, both in 
narrative tropes of automated workers consuming planets or even galaxies and in their use of very large numbers that are practically meaningless on human scales of comprehension and which can only be meaningfully altered by the game's automated processes. Clickers can thus perform a kind of auto-critique, as all video games make use of automation and numerical accumulation to some extent. Gerstmann broaches this possibility in discussing how playing clicker games heightened his awareness that most video games are already about making numbers go up; clickers just dispense with the pretense of hiding it. Along this same line of critique, Sonia Fizek focuses on the clicker's arc from active human play to passive computerized automation in her incisive look at the contradictory, "self-playing" nature of the genre. She uses Robert Pfaller and Slavoj Žižek's theories of interpassivity originally used to critique the discourse of interactivity in art, as a deconstructive tool for game studies, where the rhetoric of interactivity is nearly impossible to escape. $\underline{30}$

As the inverse of interactive media designed to compel action from human subjects, interpassive media which "supply the very process of their reception" allow for the subject to delegate or offload their pleasure to the medium itself. Using the analogy of a 19th century street barrel organ, which requires intermittent grinding but which otherwise operates automatically, Fizek argues that idle games are an interpassive form that confounds assumptions regarding interactivity:

In an idle game, the player's agency collapses in a subversive act of play delegation. The player makes an attempt to click themselves away from the responsibility of being the sole agent. Paradoxically, with every delegated click comes an enacted click of the player, and so the agency and non-agency dance in an eternally unfolding embrace... Interpassivity deconstructs interactivity-centred discourse and lays bare the illusory nature of activity. $\underline{31}$

Fizek's analysis aligns with Björk and Juul's characterization that "most zero-player games need players that interact with the games and do so with perceived intentionality. However, these players do not need to be humans to be able to fulfill these requirements." [emphasis in original] $\underline{32}$ This is a valuable reading of the clicker game, particularly in its characterization of the "dance" between agency and nonagency, which underlines a key specificity of the genre and helps explain its appeal from a design perspective. However, I believe this assessment of the genre as "depriving the player of gameplay" wherein "the player falls under the illusion of being active, while their true position, as embodied in the fetish of the self-playing idle game, remains passive," does not fully explain the player experience. $\underline{33}$ This stance, which 
regards clicker gameplay as a form of misrecognition, results from envisioning waiting as an overly negative capacity and as non-action, similar to Bogost. Under those conditions, the clicker game truly does "play itself," the time players spend away from the game is passive in an absolute sense, and the players themselves are automatized in the process of transferring their pleasure to the self-playing game.

However, the situation between player and game becomes more complicated if one characterizes waiting as active and intentional. Keogh and Richardson conclude that "it is often the real-time waiting that effectively provides players with a sense of enjoyment and a satisfying gameplay experience." $\underline{34}$ Alharthi et al. use Stephanie Boluk and Patrick LeMieux's concept of metagaming (the range of activity instantiated by games which exists "around" them) to examine the activity of waiting. They argue that "players use a range of techniques to structure the waiting periods between play sessions, during which they are neither clearly playing nor clearly not-playing. Players during these periods are metagaming by moving away from the actual game in order to seek tips from out-of-game resources, plan future moves, or express their joy or frustration from the game." $\underline{35}$ Waiting in the context of a clicker game has intentionality; the presence of the game, even if not directly apprehended by the player at a given moment, takes otherwise unmarked time and gives it a directedness which is redeemed and activated when the player returns to the dance of agency and non-agency.

The satisfaction reported by Keogh and Richardson's respondents does not come from a process of misrecognition, but instead from a substantive recognition that arises from confronting temporal structures. The player's time spent waiting has a value that becomes specific and recognizable within the context of the game, which is why microtransactions can attempt to place a price on it and why cheating to give yourself a cendoquadragintillion (10430) cookies irrevocably disrupts the experience. While some clickers might monetize their waiting systems, and some do so more exploitatively than others, all the games in this genre crystallize this time-value nexus. They stage a confrontation between the player and their conception of time, how they organize it, and how they value it. In re-centering the act of waiting as technological engagement, Jason Farman writes that "Waiting isn't essentially a wasted in-between time; instead waiting is a core part of messages we send each other across the fiber optic lines. The time it takes to receive and interpret a message is also part of its content. We take the moment of waiting and give it meaning; it becomes a message of its own." $\underline{36}$ Messages that we send and receive instantaneously have different weight 
(or wait) compared to those that take hours or days or weeks to arrive, and so it is with the messages - the numbers going up - that constitute a game. The significance of a video game experience, then, lies not just in enumerating the actions of gameplay and their effects, but also in how those actions are structured across time, and what lies in between. The notion of waiting as active gameplay, as a core part of the message, becomes clearer when the clicker game is situated back in the flows, practices, and platforms that constitute everyday life.

\section{Time Shared, Time Fragmented, Time Reclaimed}

The act of playing a clicker game exists in a cultural context wherein computer processing power is predominantly managed through personalized devices that are frequently, or in some cases continuously, within arm's reach: smartphones, laptops, and desktop computers. This individuated intersubjectivity between user and computer frames the gameworld of a clicker game and rehearses a logic of computing as a productive flow that extends through the present into the future. This is a historicized relationship, and Tung-hui Hu has argued that the conception of the computer user as a subject position arose during the 1960s transition from batch processing to timesharing computer systems, which also synchronized the divergent temporalities of human and computer. $\underline{37}$ Previously, to make use of computer power, a person would submit a set of problems or instructions that mainframes would process in batches behind a veil of secrecy, and answers would emerge some time later. The new timesharing user, on the other hand, sat in front of a terminal that seemed to promise direct, unfettered access to the processes themselves. The "time-sharing" aspect of this system aimed to maximize the efficiency of a relationship between two mismatched components operating at completely different speeds: human cognition, operating slowly and in fits and starts with comparatively long stretches of seeminglywasteful inactive contemplation, and computer processing, which can calculate at exponentially faster speeds for continuous stretches of time, unflagging-but otherwise inert without human intervention.

The time-sharing system creates immediacy from the intermittent because an aggregate of individual users can access the same system at the same time to use the flow of processing power that would otherwise go unused if only one person could connect to the system at a time. Once people perceived the computer not merely as an object but as a flow of processing-time that could constantly work and would otherwise be idle, computer users fashioned themselves as the schedulers and packagers of that time. And within this relationship, idleness became the enemy: every minute that 
passed was lost forever, and if the computer was idle, more tasks and more users were needed. Hu writes that the "real time" of the computer "actually functions as an ideology of economic productivity. By splitting a problem into thousands of increments, and then stitching these intervals of computer and worker time alike back into a seeming whole, the computer disavows unproductive moments..." $\underline{38}$ One sign that this logic of computerized productivity has become thoroughly pervasive, even extreme, manifests as the rise of cryptocurrency mining since 2008. Because Bitcoin and other copycat cryptocurrencies are accepted to have monetary value in some circles, and that value is based on the records stored on a public-yet-encrypted "blockchain" ledger, users can "mine" currency by using computers to decrypt and validate elements of the blockchain and thus earn value, albeit with increasing difficulty and requiring more intensive processing. By 2010, entrepreneurial crypto miners were using arrays of graphics processing units, originally designed for playing computer games, to mine Bitcoin-a brute-force instance of transforming processing time into potential economic profit. $\underline{39}$ Or, in other words, if one has enough computing power and time, the numbers keep going up.

Since those time-sharing days, individual management of one's computing environment at a personalized hardware-based point of access has increasingly become the norm, especially with the ascendancy of smartphones. These devices provide a continuous flow of processing time, as the 1960s time-sharing systems did, but the human-computer relationship is practically the inverse: rather than people scheduling tasks within computer-time to maximize the hardware's utilization, it is more often the case that computer applications intervene in their user's time to maximize the human's "utilization." Productivity apps of all stripes promise to reshape one's time for maximum efficiency, whether through the push notifications and alerts of to-do lists and organizers, or timers that aim to block distractions and provide milestones for completing work in discrete chunks of time. The apps that power the "sharing economy" operate on the same principles; consider the rideshare driver whose route to the next passenger has already been calculated by the app before they have even dropped off their current one. This inversion illustrates Sarah Sharma's concept of power-chronography, wherein the temporal relations of labor consist not just in selling one's time to capital, but in "the looming expectation that everyone must become an entrepreneur of time control within highly differential relationships to time." $\underline{40}$ Within these temporal inequities that valorize certain configurations of time management, such as those of the business traveler, Sharma specifically notes that 
"temporal architecture elevates the cultural significance of waiting from the dead time of doing nothing to a time of self-improvement and a privileged moment of reprieve." $\underline{41}$ The clicker genre dramatizes how waiting fits into the logic of productivity and synchronization of human-computer temporality by inserting itself into the gaps of a player's schedule; its qualities of variable, intermittent, and extensive play duration come from how it manages the time of its human environment. The synopsis of Cookie Clicker's gameplay loop creates the appearance of continuous upward progress towards larger and larger numbers, but this trajectory is constructed from a widelydispersed constellation of play moments pulled from the flow of the everyday. A player might devote several minutes of focused clicking, especially to nudge things over the threshold of a significant game milestone; or, on a particularly hectic day with other concerns, the player might take just a few seconds to check on the exponential machine and keep it moving. A player might even forget about the game for weeks, eventually coming back to it and seeing that a little progress has been made nonetheless, and the possibility always exists for more. The "idle" in idle game refers to not just the computer process left running while human attention is elsewhere, but also to the human process: to the gaps in time that are stitched together with play. With these structures, clicker games are an example of what Sharma calls "novel temporal experiences" designed to produce a sense of meaningfulness within larger temporal architectures of time maintenance. $\underline{42}$ 


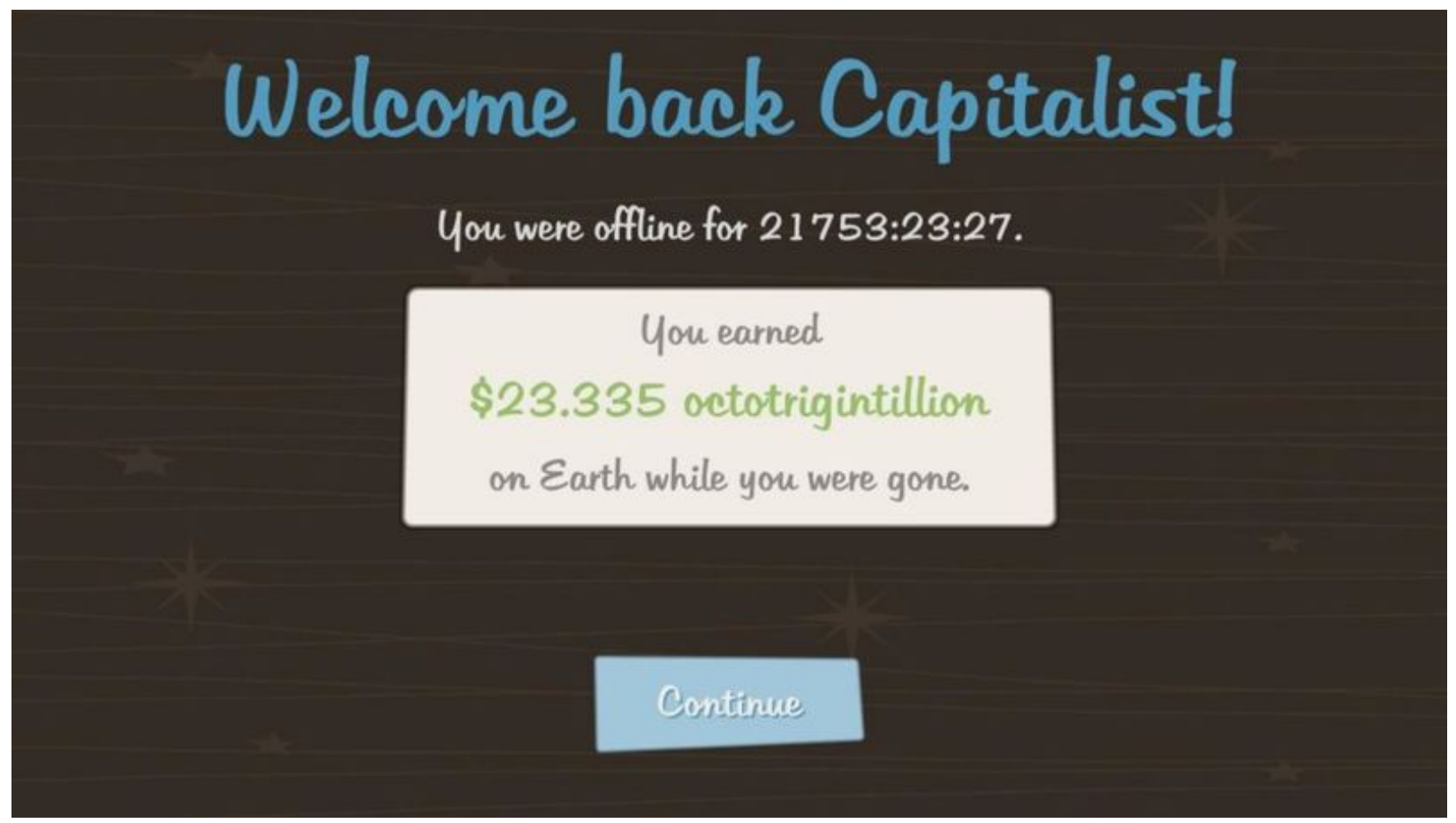

Figure 5. Hyper Hippo, AdVenture Capitalist, 2014, video game.

In any case, this linkage between human and computer temporalities helps explain why clicker games are designed mainly for personal computers and phones and are nearly absent from home game consoles. While initiatives such as the Folding@home project for the PlayStation 3 have used the idle processing power of consoles for research computing, idle gameplay as such is more likely to appear as a minor ancillary element within larger games. There have certainly been a few ports of clicker games such as AdVenture Capitalist, originally developed for web browsers in 2014 and eventually released on the PlayStation 4 console in 2016 (figure 5). But the game console framework inherits a televisual legacy and conventionally presupposes a single game program loaded into memory and a player sitting in front of a television devoting sustained attention to that program. Both these assumptions fit poorly with the gameplay aesthetics of clickers. The console's processing power to run the game, and the player's effort to sit down and play, would be "wasted" if singularly devoted to such a sparse pattern of gameplay action. Clickers instead demand multitasking environments. They live in the minimized windows of desktops and run in the background of phones and get pushed onto secondary browser tabs, intermittently surging to the foreground when the right time comes. They also expect that their players are to some extent tethered to a device that they can easily retrieve, click on a few times, and just as easily put away. 
Clicker games thus even more directly chart the technocultural territory that Murray identified with Tetris; the genre is a symptom of the technocratic neoliberal regime of time that creates the overtasked lives and overcrowded schedules of the present. Yet in its aesthetic provocations, the clicker also allows for symbolically experiencing agency and holds the possibility for that agency to extend beyond the symbolic. On one hand, clicker games easily slip into the flexible, fragmented, and fungible regime of time enabled by ubiquitous computing; in fact, these games actually require it to be viable forms of play. Jonathan Crary's concept of 24/7 capitalism rests on a neoliberal colonization of time that has erased "distinctions between work and non-work time, between public and private, between everyday life and organized institutional milieus." $\underline{43}$ By making legible ever more granular segments of time and exerting control over them, contemporary computing has greatly accelerated this erasure of distinctions. After all, the ethos driving the digitized casualization of labor and the socalled "sharing" economy is about convincing us that cars and housing are not things but flows of productivity that can also be liberated from idleness if we digitally reconfigure our own temporalities. The worst clicker games rehearse these impulses when they activate the psychology of addiction and conflate time and money to exploit players and extract value from them, but all games in the genre rely on this reconfigured temporality, as their minimized gameplay flows into even the minutest fragments of time. Without those fragments, with only the monotasking and sustained duration typified by console gameplay, clickers might not exist. 


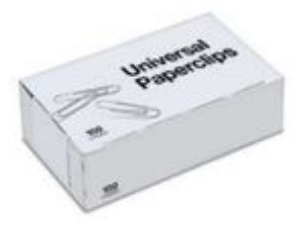

\section{Figure 6. Frank Lantz, Universal Paperclips, 2017, video game.}

But even as the clicker genre occupies these dispersed units of time within players' lives, it leaves open myriad possibilities for what to do with that time. Some clickers in their narrative and aesthetic strategies aim to critique the modalities that gave rise to their making. Frank Lantz's web-based Universal Paperclips (2017), for example, uses the Cookie Clicker template but trades cookie production for paperclip production, and adds a narrative arc inspired by thought experiments on runaway artificial intelligence (figure 6). Paolo Pedercini, who has an interest in "the wicked problem of the duration of play experiences," highlights Universal Paperclips as a game that artfully "wastes time." As a clicker game, it acts through the time-value nexus, but Pedercini praises the game's "capacity to gradually morph its internal economy. While the end remains the same, the means expand and transform continuously. The gameplay gradually incorporates marketing, soft power, and finance, and eventually goes through major paradigm shifts. It's a remarkable dramatization of the different phases of capitalism, albeit without explicit mention of crisis." $\underline{44}$ In a genre characterized by endless cyclical growth, the ending of Universal Paperclips offers a measure of permanent closure. The game casts the player as an artificial intelligence tasked with manufacturing paperclips, and the exponential machine by the end of the game has transformed nearly all matter in the universe into clips. At this point, the player can choose to reset the timeline and start from 0 clips; the reset is a typical gameplay mechanic for many clickers. But players can instead choose to press on, and end with a universe comprised of nothing but 30 septendecillion (3054) paperclips. This ending is 
permanent insofar as there is no way to reset this within the game, and every time the player returns to the game's website, the game state remains the same. This irrevocable permanence presents one possibility for confounding the perpetual expansion at the heart of the genre's dramatization of temporality-at least, until one goes outside the game and deletes their browsing data. $\underline{45}$

But beyond specific instances of pointed critique, what might we make of the clicker genre as a whole and how it uses duration? In video games, genre is partly the result of a collective conversation about the value of a specific mode of gameplay. A certain game aesthetic speaks to players and to designers who rehearse and reiterate that aesthetic amidst a host of considerations about how players will approach it. One of those considerations must be the temporality of everyday life and the minutes and hours within which a person can be a player. As a genre, clickers engage some of both the shortest units of playtime and the longest, and to gain the fullest sense of the significance of those aesthetic qualities, it is worth revisiting the comparison with walking simulators from a different angle. The walking sim genre proved so incendiary to some because by winnowing away most vestiges of what that segment of the audience considered "real" gameplay, it also eliminated recourse to long-established frameworks that games use to conceive of navigable space: as a battlefield to be won, as a puzzle to be solved, as a landscape to be occupied. $\underline{46}$ By eliminating those familiar markers without replacing them, walking sims offer the possibility of contemplating and perceiving space differently.

The clicker genre offers a similar possibility for time. Characterizing what occurs in clickers as a lack of gameplay or as not playing at all is to fall back on familiar temporal conceptions of gameplay and recoil at their absence-the idea of play as a delimited leisure activity with a legible beginning and end, for example. For casual games, Anable rejects such characterizations to highlight the interstitial qualities of those "rhythmic interludes" where "clicks also create an expressive relationship across modes (work/play), spaces (the place of work/elsewhere), and bodies (the player's body/the computer's body and the wider community of players." $\underline{47}$ As a subset of games within the casual framework, clicker games sharpen that notion of the click and expand the expressiveness of not clicking across temporal interludes. Clicker games ride on the continuous and ubiquitous flow of computing-time that structures most of everyday life, making the conflationary logic of the time-value nexus visible and thus offering the first step to challenging it. By encouraging the player to wait with purpose and to conceive of waiting as an active state, the genre suggests that time spent idling 
is not wasted or lost, as the computerized ideology of economic productivity might have us believe. And by leading the player in a dance between agency and non-agency on a gossamer strand of action for such a long duration as to seem absurd, these games allow the player the chance to surmise that maybe there are other aspects of the temporal structures that govern our lives that are just as absurd-clickers just dispense with the pretense of hiding it.

$* * *$

Oscar Moralde is a doctoral candidate in Cinema and Media Studies at UCLA. He studies embodied aesthetics and ideology in video games and other media. His writing can be found in The Journal of Popular Culture, Well Played Journal, Media Fields Journal, and the Criterion Collection.

\section{Footnotes}

1. Aristotle, Physics IV.12, 221a-b, quoted in Charles E. Scott, The Time of Memory: Teachers and the Role of the Teachers' Lounge (Albany: SUNY Press, 1999), 291. 2. Julien "Orteil" Thiennot, Cookie Clicker, 2013, video game. $\subseteq$ 3. See Graeme Kirkpatrick, Aesthetic Theory and the Video Game (Manchester: Manchester UP, 2011); Brendan Keogh, A Play of Bodies: How We Perceive Videogames (Cambridge, MA: MIT Press, 2018); Aubrey Anable, Playing with Feelings: Video Games and Affect (Minneapolis: University of Minnesota Press, 2018). $\underline{\underline{ }}$

4. Janet Murray, Hamlet on the Holodeck: The Future of Narrative in Cyberspace, updated ed. (Cambridge, MA: MIT Press, 2017), 143-144.

5. See Markku Eskelinen, "The gaming situation," Game Studies 1, no. 1 (2001), http://gamestudies.org/0101/eskelinen/; Celia Pearce, "Theory Wars: An Argument Against Arguments in the So-Called Ludology/Narratology Debate," Proceedings of the 2005 DiGRA International Conference: Changing Views: Worlds in Play, Vancouver, Canada, 16-20 June 2005, http://www.digra.org/wpcontent/uploads/digital-library/06278.03452.pdf; Murray, Hamlet on the Holodeck, 178. $ヒ$ 
6. See Jason Mittell, Complex TV: The Poetics of Contemporary Television Storytelling (New York: NYU Press, 2015); Rachel E. Silverman and Emily D. Ryalls, “'Everything is Different the Second Time Around': The Stigma of Temporality on Orange is the New Black," Television and New Media 17, no. 6 (September 2016): 520-533; Tiago de Luca, "Slow Time, Visible Cinema: Duration, Experience and Spectatorship," Cinema Journal 56, no. 1 (Winter 2016): 23-42.

7. Steven Neale, "Questions of Genre," in Film and Theory: An Anthology, eds. Robert Stam and Toby Miller (Oxford: Wiley-Blackwell, 2000), 157-178; Linda Williams, "Film Bodies: Gender, Genre, and Excess," Film Quarterly 44, no. 4 (Summer 1991): 2-13.

8. Thomas H. Apperley, "Genre and game studies: Toward a critical approach to video game genres," Simulation \& Gaming 37, no. 1 (March 2006): 7; Dominic Arsenault, "Video Game Genre, Evolution, and Innovation," Eludamos: Journal for Computer Game Culture 3, no. 2 (2009): 171. $\_$

9. Neale, "Questions of Genre," 165-166; Arsenault, "Video Game Genre," 162. 10. Christopher Hanson, Game Time: Understanding Temporality in Video Games (Bloomington: Indiana UP, 2018), 2. Also see Brenda Kay Laurel, Computers as Theatre, $2^{\text {nd }}$ Ed. (Reading, MA: Addison-Wesley, 2013); Jesper Juul, Half-Real: Video Games between Real Rules and Fictional Worlds (Cambridge, MA: MIT Press, 2005); Michael Nitsche, "Mapping Time in Video Games," in Situated Play: Proceedings of the Third International Conference of the Digital Games Research Association DiGRA '07, ed. Akira Baba (Tokyo: University of Tokyo, 2007), 145-151; David O'Grady, "Movies in the Gameworld: Revisiting the Video Game Cutscene and its Temporal Implications," in The Game Culture Reader, eds. Jason C. Thompson and Marc A. Ouellette (Newcastle upon Tyne: Cambridge Scholars Publishing, 2013), 103-124.

11. Hanson, Game Time, 195. $\_$

12. Jeff Gerstmann, “Giant Bomb’s Game of the Year 2015: Day Five,” Giant Bomb, podcast audio, 1 January 2016, https://www.giantbomb.com/podcasts/giantbombs-game-of-the-year-2015-day-five/1600-1452/.

13. Brendan Keogh and Ingrid Richardson, "Waiting to play: the labour of background games," European Journal of Cultural Studies 21, no. 1 (2018):15-20. 
14. Sultan A. Alharthi, et al., The Pleasure of Playing Less: A Study of Incremental Games through the Lens of Kittens (Pittsburgh: Carnegie Mellon University ETC Press, 2017), 5.

15. Alharthi, et al., Pleasure of Playing Less, $6 . \leftrightharpoons$

16. Nathan Grayson, "Clicker Games Are Suddenly Everywhere on Steam," Steamed Kotaku, 30 July 2015, https://kotaku.com/clicker-games-are-suddenly-everywhere-onsteam-1721131416.

17. Alharthi et al., Pleasure of Playing Less, $2 . \bullet$

18. Melissa Kagen, "Walking Simulators, \#GamerGate, and the Gender of Wandering," in The Year's Work in Nerds, Wonks, and Neocons, eds. Jonathan Paul Eburne and Benjamin Schreier (Bloomington: Indiana UP, 2017), 288. 19. Kill Screen Staff, “Is it Time to Stop Using the Term 'Walking Simulator'?” Kill Screen, 30 September 2016, https://killscreen.com/articles/time-stop-using-termwalking-simulator/; Danielle Riendeau, "Walking Simulators are growing up and stepping out," ZAM, 2 August 2016, http://readyset.zam.com/article/865/walking= simulators-are-growing-up-and-stepping-out/. $\_$ 20. Anable, Playing with Feelings, 84. Also see John Vanderhoef, "Casual Threats: The Feminization of Casual Video Games," Ada: A Journal of Gender, New Media, and Technology, no. 2 (June 2013), https://adanewmedia.org/2013/06/issue2-vanderhoef/.

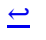

21. Staffan Björk and Jesper Juul. "Zero-Player Games-Or: What We Talk about When We Talk about Players." In The Philosophy of Computer Games Conference. Madrid, 2012. http://www.jesperjuul.net/text/zeroplayergames/. 22. Jason Tanz, "The Curse of Cow Clicker. How a Cheeky Satire Became a Videogame Hit." Wired Magazine, 20 December 2011, https://www.wired.com/2011/12/ff_cowclicker/all/1/. 23. Ian Bogost, Play Anything: The Pleasure of Limits, the Uses of Boredom and the Secret of Games (New York: Basic Books, 2016), 208-9. $\_$ 24. Bogost, Play Anything, 210. 
25. Alec Meer, "My Addiction to AdVenture Capitalist: An Update," Rock Paper Shotgun, 9 August 2015, https://www.rockpapershotgun.com/2015/08/09/my= addiction-to-adventure-capitalist-an-update/; Matt Wales, "Clicker Heroes studio abandons upcoming sequel's free-to-play model for 'ethical reasons'”, Eurogamer, 20 November 2017, https://www.eurogamer.net/articles/2017-11-20-clicker-heroesstudio-abandons-upcoming-sequels-free-to-play-model-for-ethical-reasons/.. 26. Keogh and Richardson, "Waiting to Play," 15. 27. Bogost, Play Anything, 206-207. 28. Keogh and Richardson, "Waiting to Play," 22. 29. Keogh and Richardson, "Waiting to Play," 14. 30. Sonia Fizek, "Interpassivity and the Joy of Delegated Play in Idle Games," Transactions of the Digital Games Research Association 3, no. 3 (April 2018): 151. https://doi.org/10.26503/todigra.v3i3.81.

31. Fizek, "Interpassivity," 153. 32. Björk and Juul, "Zero-Player Games." $\triangleq$

33. Fizek, "Interpassivity," 148.

34. Keogh and Richardson, "Waiting to Play," 22. 35. Alharthi et al., Pleasure of Playing Less, 37. Also see Stephanie Boluk and Patrick LeMieux, Metagaming: Playing, Competing, Spectating, Cheating, Trading, Making, and Breaking Videogames (Minneapolis, Minn.: University of Minnesota Press, 2017). $\leftrightarrows$

36. Jason Farman, “Fidget Spinners,” Real Life, June 28, 2017, https://reallifemag.com/fidget-spinners/. 37. Tung-Hui Hu, A Prehistory of the Cloud (Cambridge, MA: MIT Press, 2015), 43. 38. Hu, Prehistory of the Cloud, $47 . \in$

39. Lana Swartz, "What was Bitcoin, what will it be?

The technoeconomic imaginaries of a new money technology," Cultural Studies 32 no. 4 (2018):635, DOI: 10.1080/09502386.2017.1416420 
40. Sarah Sharma, In the Meantime: Temporality and Cultural Politics (Durham: Duke University Press, 2014), 138.

41. Sharma, In the Meantime, 53.

42. Sharma, In the Meantime, 139.

43. Jonathan Crary, 24/7: Late Capitalism and the Ends of Sleep (London: Verso, 2013), 74.

44. Paolo Pedercini, “Top 2017 Games That Waste Your Time Properly," Molleindustria (blog), 27 December 2017, http://www.molleindustria.org/blog/top-2017-games-that-waste-your-time-properly $1 . \pm$

45. Colloquially, deleting their cookies. $\doteq$

46. Oscar Moralde, "Haptic Landscapes: Dear Esther and Embodied Video Game Space," Media Fields Journal, no. 8 (2014), http://mediafieldsjournal.org/hapticlandscapes $/ . \doteq$

47. Anable, Playing with Feelings, $97 . \longleftarrow$ 